\title{
Urodynamic Patterns of the Idiopathic Overactive Bladder
}

\author{
Yuriy M. Dekhtiar, Fedir I. Kostyev, Oleksandr V. Rudenko, Dmytro O. Kuznietsov
}

Department of Urology and Nephrology, Odessa National Medical University, Odessa, Ukraine

\section{Email address:}

kuznetsovmake@gmail.com (D. O. Kuznietsov)

\section{To cite this article:}

Yuriy M. Dekhtiar, Fedir I. Kostyev, Oleksandr V. Rudenko, Dmytro O. Kuznietsov. Urodynamic Patterns of the Idiopathic Overactive Bladder. Science Journal of Clinical Medicine. Vol. 6, No. 5, 2017, pp. 74-79. doi: 10.11648/j.sjcm.20170605.12

Received: August 16, 2017; Accepted: September 4, 2017; Published: October 19, 2017

\begin{abstract}
The aim of study was the investigation of frequency and clinical significance of selected stereotypical recurring urodynamic patterns, which are manifested by the results of a complex urodynamic study in patients with idiopathic overactive bladder. The combined urodynamic study (CUS) was conducted in 137 patients with IOAB. The standard cystotonometry allowed to observe the maximum cystometric capacity. It also allowed to determine the bladder sensitivity (BS) and detect the presence of detrusor overactivity (DO). The results of retrograde cystotonometry established three variants of detrusor overactivity: phase DO, terminal DO, and stress-induced DO. The study of the bladder function using methods of UFM and EMG of anal sphincter in the emptying phase, confirmed the association of obstructive urination with incomplete discharge of the urethra from the effects of the sphincter. The coordination of function of the detrusor and external sphincter were assessed according to the results of CUS and sphincteric EMG. The EMG analysis in Work / Rest Assessment mode allowed to reveal the characteristic changes in the biopotential indices of the pelvic sphincter and indicated their correlation with the clinical features of the disease course. The imperative character of urination was established in 137 patients with IOAB during CUS characterized by hyperactivity and hypertensity of bladder, depending on the severity of the violation of the integrative function of LUT.
\end{abstract}

Keywords: Overactive Bladder, Cystotonometry, Uroflowmetry, Detrusor Overactivity

\section{Introduction}

Overactive Bladder (OAB), also known as Overactive Bladder Syndrome, is a condition of frequent feel to urinate which adversely affects the quality of life. Frequent urges for urination may occur at the day, at night, or both, with urinary incontinence (UI) or without it [1]. Although the overall prevalence of $\mathrm{OAB}$ is similar between men and women, there are sex-specific differences in the prevalence of various symptoms within the $\mathrm{OAB}$ complex [2-4]. Anatomical and physiological differences in the lower urinary tract of males and females may help to explain these variations. Overall, $\mathrm{OAB}$ prevalence rates in large population-based studies range from $7-27 \%$ in men and $9-43 \%$ in women. A proportion of $\mathrm{OAB}$ cases $(37-39 \%)$ has correlated during a given year, but the majority of patients had persistent symptoms [5-6].

Diagnostics of the lower urinary tract symptoms (LUTS) in patients with idiopathic overactive bladder (IOAB) consists of complaints, anamnesis, neurological and urological examinations. It also requires the additional survey methods, among which the urodynamic testing occupies a major place. The discussion about clinical significance of the urodynamic tests in the diagnostics of various forms of urination disorders is still relevant [1,7]. However, the opinions on this matter are greatly contrasting. Some researchers believe that the urodynamics are mandatory for diagnosis and treatment among patients with $\mathrm{OAB}$ symptoms, because diagnosis based on urinary symptoms alone, would lead to under-diagnosis of detrusor overactivity (DO) [8].

The cause of IOAB might be detrusor overactivity, in which the bladder muscle (the detrusor) contracts unexpectedly during bladder filling. Urodynamics can be used to confirm the presence of detrusor overactivity, which may help guide treatment. An overactive detrusor can be associated with urge incontinence [9].

The two main aims in study of the lower urinary tract urodynamics are: to reproduce symptomatic complaints of a patient during urodynamic tests, and to provide a pathophysiological explanation by comparing the patient's symptoms with the results of urodynamic studies. There is an 
opinion that LUTS is important because it leads the patient to a doctor, but it also complicates the treatment tactics for the doctor [10].

\section{Purpose of the Study}

The purpose of the study was to determine the frequency and clinical significance of selected stereotypical recurring urodynamic patterns, which are manifested by the results of urodynamic studies in patients with idiopathic overactive bladder.

\section{Method}

The combined urodynamic studies (CUS) (uroflowmetry, urethral profilometry, filling and emptying cystotonometry in the "pressure-flow study") was conducted in 137 patients (105 women, 32 males) with IOAB. The study was conducted on the urodynamic device "Pico Compact" (Menfis Bio Medica, Italy) and Delphis KT (Laborie, Canada), according to the recommendations of the International Committee for the Prevention of Urinary Incontinence (UI). The study of the pelvic floor muscle system bioelectric activity with computer electromyography (EMG) of the sphincter pelvic apparatus was conducted on a 2-channel electromyograph "Neuro Trac ${ }^{\mathrm{TM}}$ Myo Plus 4" in the Work / Rest Assessment mode.

\section{Result}

The standard cystotonometry allowed to observe the maximum cystometric capacity. It also allowed to determine the bladder sensitivity (BS) and detect the presence of detrusor overactivity (DO) (Table 1).

Bladder sensitivity is the only subjective parameter whose assessment only depends on the patient. The bladder sensitivity is determined during retrograde cystotonometry and closely related to the volume and rate of fluid intake during the study. The increased sensitivity of bladder (hypersensitivity) was defined as urge to urinate, which arised early and persisted until the end of the study.

Table 1. Frequency of urodynamic symptoms in patients with IOAB $(n=$ 137).

\begin{tabular}{ll}
\hline Symptoms & Number of patients (\%) \\
\hline Hypersensitivity & $97(70,8 \%)$ \\
Decrease of compliance & $59(43,1 \%)$ \\
Detrusor overactivity & $49(35,8 \%)$ \\
Urinary incontinence during the study & $31(22,6 \%)$ \\
Functional intravesical obstruction & $47(34,3 \%)$ \\
Sphincter insufficiency & $61(44,5 \%)$ \\
Reduction of the functional urethral length & $55(40,1 \%)$ \\
Dysfunctional urination (pseudodysynergia) & $10(7,3 \%)$ \\
Instability of urethra & $34(24,8 \%)$ \\
Cystalgias during the study & $37(27 \%)$ \\
Reduction of maximum cystometric capacity & $118(86,1 \%)$ \\
\hline
\end{tabular}

The results of retrograde cystotonometry established three variants of detrusor overactivity: phase DO, terminal DO, and stress-induced DO. The phase DO was defined as the presence of phase waves with urine leakage or without it. The terminal DO was defined as the uninhibited reduction of detrusor. Phase DO was the most frequently observed in younger patients, regardless of gender. The average age of women with phase DO was 41.5 years. The average age of women with terminal DO was 62.3 years. The average age of males with phase DO was 43.3 years and 58.1 years in males with terminal DO.

The volume of bladder with one uninhibited reduction of the detrusor was greater in phase DO $(117.3 \pm 11.7 \mathrm{ml}$ versus $83.8 \pm 9.1 \mathrm{ml})$. Other parameters did not differ significantly in both genders. The amplitude of one uninhibited reduction of the detrusor during the phase DO was significantly lower than terminal DO in both genders.

Phase detrusor overactivity is a wave-like involuntary reduction of the detrusor, which often leads to the urgency (Figure 1).

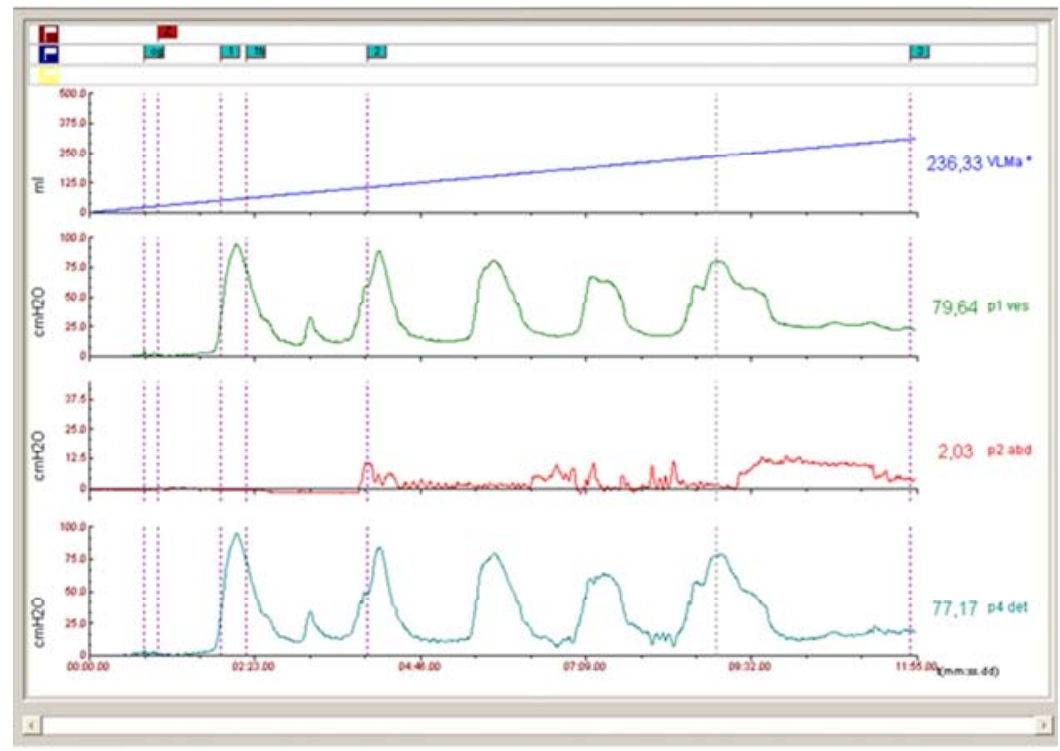

Figure 1. Retrograde cystotomometry of the patient E., 36 years old. Phase DO, wavy involuntary reduction of detrusor. 
The phase reduction of the detrusor may not be accompanied by certain feelings of the patient. It can be perceived as the first sensation of bladder filling, or standard urge to urinate. Terminal DO was characterized by the presence of a single involuntary reduction of the detrusor, which occurred when the bladder filled to the maximum cystometric capacity (Figure 2, Figure 3). Stress-induced DO is a significant increase in intravesical pressure, occurred due to physical effort, coughing, sneezing, or change in body position (Figure 4).

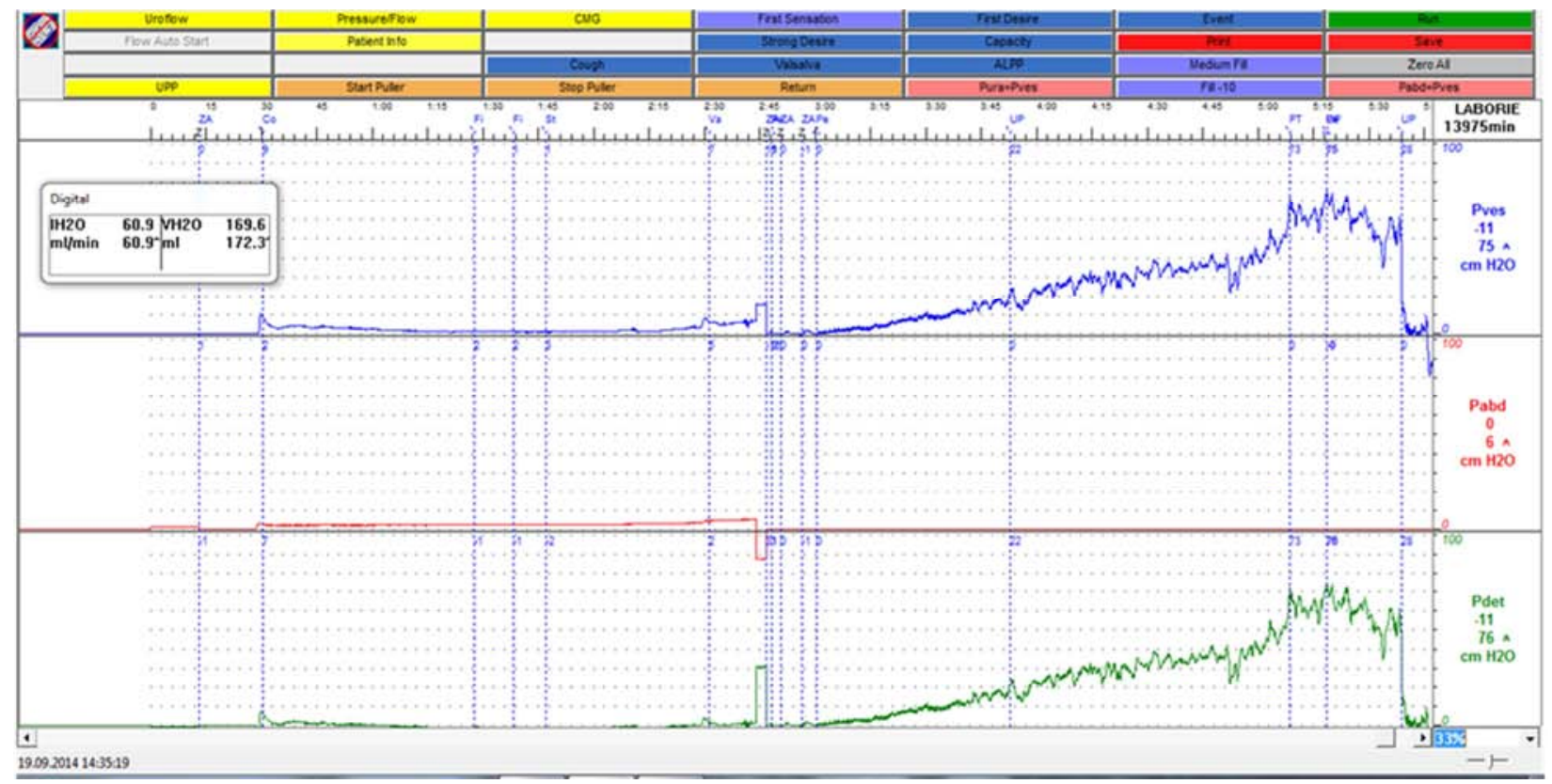

Figure 2. Retrograde cystotomometry of patient $S$., 34 years. Terminal DO.

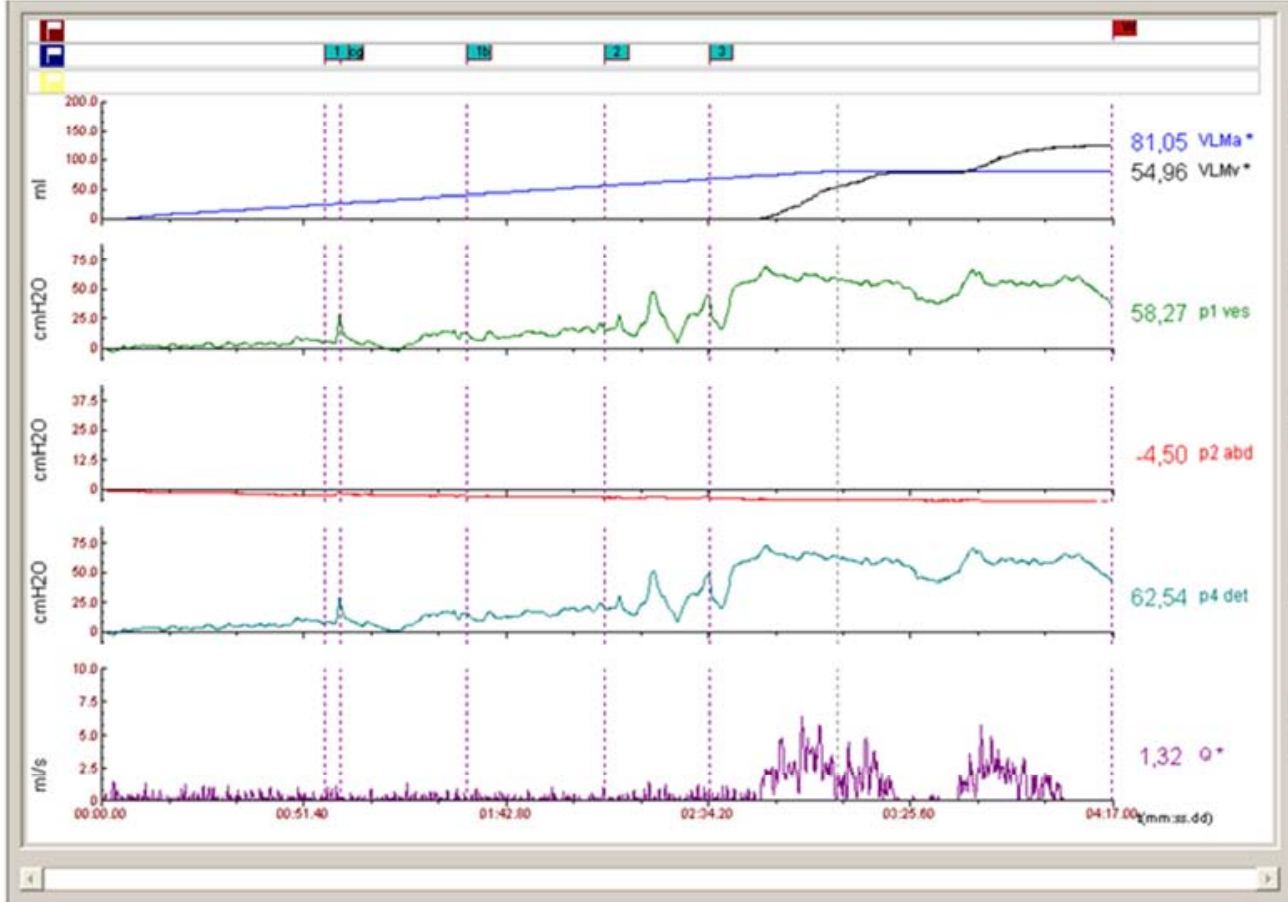

Figure 3. Investigation of pressure-flow." Patient L., 67 years old. The phase of the terminal DO with urine loss (urgent incontinence).

One of the most important functional lower urinary tract indicators is the extensiveness (compliance) of the bladder. Bladder compliance states the communication between the change in volume of bladder filling and the increase of detrusor pressure during the CUS. Bladder compliance is the ability to maintain stable indices of intravesical pressure during its filling. Its parameter is $>30-40 \mathrm{ml} / \mathrm{cm} \mathrm{H} \mathrm{H}_{2} \mathrm{O}$ in normal. It is important to remember that the pathological values of compliance can be an artifact when the bladder is filled to fast. The test of the filling speed changing was 
performed to prove it. This phenomenon was considered as an artifact, in the case of compliance normalization.

Reduction of the bladder compliance during CUS can be also considered as urodynamic pattern of the IOAB.

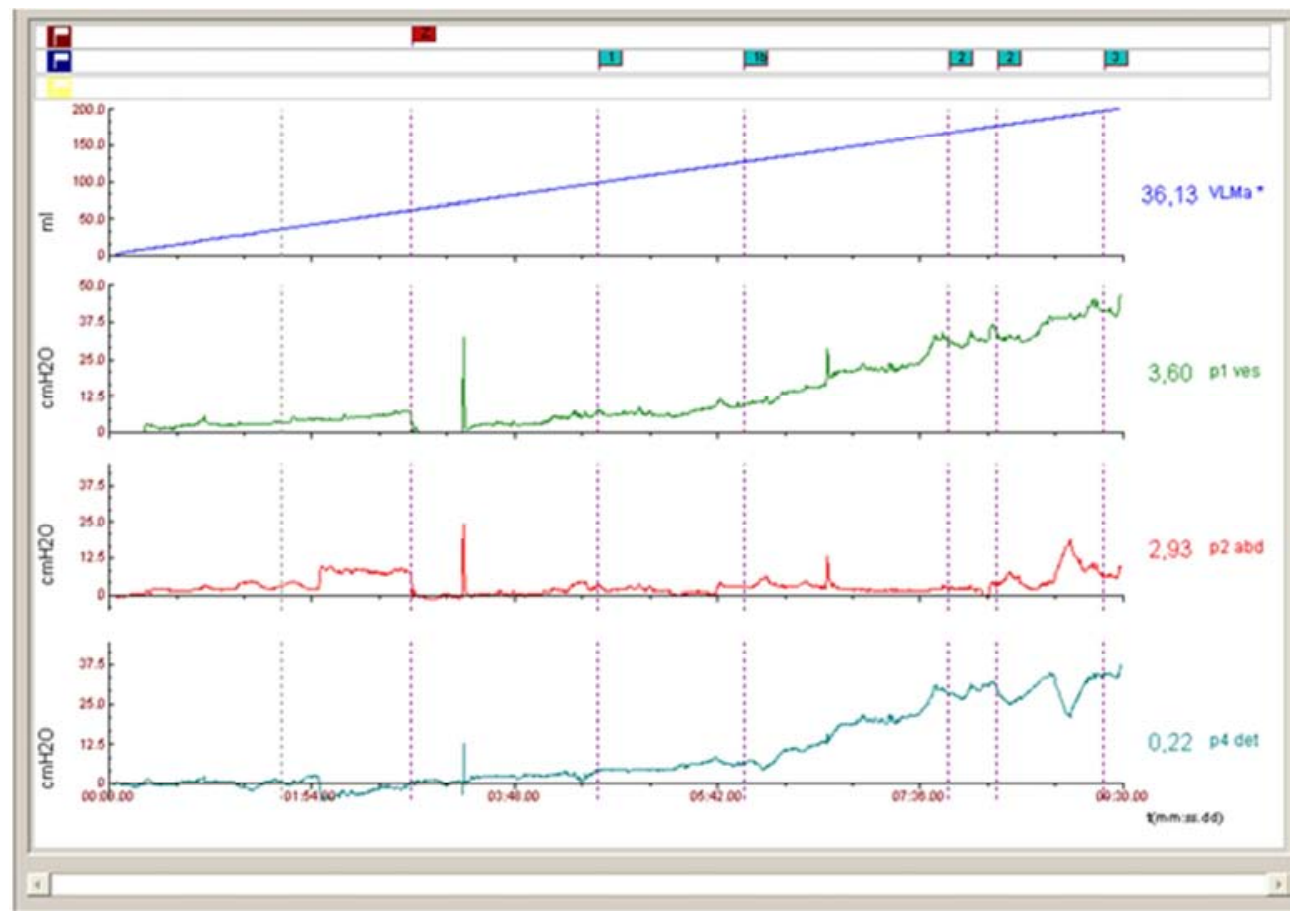

Figure 4. Retrograde cystotomometry of patient V., 41 years. Stress-induced DO detected after cough test.

Indicators of CUS may not show the true anatomical capacity of bladder, but such urodynamic indicator exceeded the volume of urinations, captured by patients in urination diaries. Normal indices of cystotomometry in our study were found in $22(15.3 \%)$ patients. At the same time, we did not exclude these patients from the treatment group of IOAB.

The function of the sphincter apparatus was suffered also. The maximum urethral pressure did not exceed $30 \mathrm{~cm}$ of water column, according to the results of urethral profilometry in $61(44.5 \%)$ patient with $\mathrm{IOAB}$, testifying to the presence of urethral sphincter deficiency. A decrease in the functional length of the urethra was detected in 55 $(40.1 \%)$ patients. The fluctuations of maximum intravesical pressure, with a static position of the sensor for 5-10 minutes and the amplitude of 15-20 cm of water column was defined as the instability of the urethra in $34(24.8 \%)$ patients. Fluctuations in maximum urethral pressure may lead to urgency and also to obstructive urination. The instability of the urethra was registered in combination with the DO and without it. Urethral instability was characterized by significant, spontaneous, non-induced deviations of urethral pressure, provided by a critical reduction in the value of closure pressure, which may cause the involuntary loss of urine due to a negative urethral-bladder pressure gradient.

The 3 probable variants of bladder excretion were observed according to the results of uroflowmetry (UFM). The normal urination was observed in $56(40.1 \%)$ patients, rapid urination in $34(24.8 \%)$ patients and obstructive urination in $47(34.3 \%)$ patients. The intermittent urination was observed in $24(17.5 \%)$ patients. An increase in the tone of the detrusor before the urination, according to results of
CUS, was observed in most of patients with IOAB. The hope for "rapid" urination was observed in the case of a proportional tone of an increase in the effective diameter of the urethra. However, it was only in 47 patients. The absence of it in other patients was due to the disproportionate opening of the sphincter apparatus. The normal micturition was observed in case of the minimal incomplete relaxation of sphincter during the urination.

The obstructive urination with functional disorders is the one of manifestations in disorders of urine evacuation detected in patients with OAB. The instability of the urethra, incomplete relaxation of the bladder neck or sphincter during micturation, pseudodyssynergia of the detrusor-sphincter segment, are the main causes of obstructive urination development in patients with OAB. Difficulty urinating in patients with $\mathrm{OAB}$ may be a consequence of a violation of bladder contractile ability, through the DO. Difficulty urinating in women with $\mathrm{OAB}$ may be also due to omission of the vaginal walls, the formation of cystocele and violation of the angle of the urethrovesical segment.

\section{Discussion}

The study of the bladder function using methods of UFM and EMG of anal sphincter in the emptying phase, confirmed the association of obstructive urination with incomplete discharge of the urethra from the effects of the sphincter. The excretion of bladder proceeds against a background of its high electrical activity. It was manifested by the registration of "outbreaks" in contractile activity of the transversalstriated sphincter of urethra and muscles of the pelvic floor, 
making it difficult to urinate or completely stopped the urinary flow.

The detailed assessment of urination in "pressure-flow" regime was found the decrease of urethral pressure during urination, which caused intravesical obstruction in patients with signs of obstructive urination and instability of the urethra. The spontaneous increase and decrease of detrusor pressure during micturation were recorded in patients with DO. It could also cause a combination of pollakiuria, urge UI and obstructive urination.

The coordination of function of the detrusor and external sphincter were assessed according to the results of CUS and sphincteric EMG. The symptoms of muscle dysfunction of the perineum and sphincter apparatus were not detected by EMG in $17.8 \%$ of patients with IOAB. The increased EMG activity of the LUT sphincter with the maximum increase of EMG signals at the time of the first urge to urinate was observed in these patients during the phase of urinary accumulation in the bladder. The arbitrary reduction of the detrusor and relaxation of the periurethral striated muscle were observed during the evacuation phase of urination. It was manifested in the sphincter EMG curve by the disappearance of electrical signals.

The EMG analysis in Work / Rest Assessment mode allowed to reveal the characteristic changes in the biopotential indices of the pelvic sphincter and indicated their correlation with the clinical features of the disease course. The symptoms of dysfunction of the perineal striated muscles and sphincter apparatus of pelvic organs were detected by the EMG study in $78.2 \%$ of women with an urgent form UI. The dysfunction of perineal muscles and sphincter apparatus was detected in $36.8 \%$ women with "sensory" symptoms of IOAB, without urinary incontinence. The same dysfunction was detected in $53.4 \%$ of patients with IOAB with pain syndrome. Symptoms of dysfunction are manifested by the contraction of the external sphincter of bladder with a cystometric determined reflex of the detrusor reduction, which is characterized by loss of ability to reduce or relax the external sphincter during the period of detrusor reduction.

The characteristic feature of EMG in the Work / Rest Assessment (62\%) mode, conducted in patients with urodynamically proven DO, was the decrease in the amplitude of biopotentials of perineal striated muscles and the increase of the biopotentials of the sphincter apparatus of the pelvic organs. The spontaneous activity of the biopotentials in striated muscles of the bladder external sphincter and the sphincter of the anus was detected in 6 $(12.2 \%)$ patients with DO in the state of relative physiological rest, during the course of CUS. It was observed on EMG curve by increasing the amplitude of the signals up to $100 \mu \mathrm{V}$ during the period of urine accumulation with imperative urges, coughing and sneezing.

Meanwhile, the urinary disorders are not primarily associated with dysfunction of the bladder external sphincter in the IOAB. It is confirmed by the absence of reliable differences in the characteristics of the EMG-curves, which analyzed in the Work / Rest Assessment mode, including the amplitude, duration of one EMG signal and the number of waves per unit time. Thus, sphincter EMG in the Work / Rest Assessment mode with sphincter EMG during urodynamic tests allows study the complete information about the functional state of LUT. The sphincter EMG acquires special significance in patients with IOAB and intravesical obstruction with dysfunction of the bladder sphincter apparatus.

The EMG study, conducted on two channels of biofeedback with the study of the intravesical pressure dynamics, was established the distinct functional state of bladder external and anal sphincter in 35 patients with IOAB. It was manifested by a significant difference in the levels of electrical activity of these sphincters. This form of dysfunction was noted in 12 patients $(24.5 \%)$ with DO and 23 patients $(26.1 \%)$ without DO.

\section{Conclusion}

1. The imperative character of urination was established in 137 patients with IOAB during CUS characterized by overactivity and hypertensity of bladder, depending on the severity of the violation of the integrative function of LUT. It leads to decrease of the maximum cystometric capacity in $86.1 \%$ of patients, decrease of the bladder sensitivity in $70,8 \%$ of patients, detrusor overactivity in $35,8 \%$ of patients, decrease of complicacy in $43,1 \%$ of patients and instability of urethra in $24,8 \%$ of patients.

2. The sphincter EMG in the Work / Rest Assessment mode during CUS allows to get more complete information about the functional state of LUT and plays an important role in the diagnosis of electro neurophysiological disorders of the pelvic floor muscle and pelvic sphincter function, changes in intravesical pressure, which is accompanied by significant differences in characteristics of EMG - curves analyzed in the Work / Rest Assessment mode and a significant reduction in the time keeping maximum muscular effort (reduction). The sphincter EMG takes significant part in observation of patients with IOAB and intravesical obstruction due to dysfunction of the bladder sphincter apparatus.

3. Reduction of the bladder ability to accumulate and retain urine at normal or low intravesical pressure and frequent combination of bladder instability with signs of obstructive urination are the main features of urodynamic violations of the lower urinary tract in patients with IOAB.
Abbreviation
IOAB
BS
DO
UFM
EMG
CUS
idiopathic overactive bladder
bladder sensitivity
detrusor overactivity
uroflowmetry
electromyography
LUTS lower urinary tract symptoms 


\section{References}

[1] Gormley EA, Lightner DJ, Burgio KL, Chai TC, Clemens JQ, Culkin DJ, Das AK, Foster Jr HE, Scarpero HM, Tessier CD, Vasavada SP. Diagnosis and treatment of overactive bladder (non-neurogenic) in adults: AUA/SUFU guideline. J Urol. 2012; 188(6): 2455-63.

[2] Vaughan CP, Johnson TM, 2nd, Ala-Lipasti MA, Cartwright R, Tammela TL, Taari K, et al. The Prevalence of Clinically Meaningful Overactive Bladder: Bother and Quality of Life Results from the Population-Based FINNO Study. Eur Urol 2011; Jan 25.

[3] Link CL, Steers WD, Kusek JW, et al. The association of adiposity and overactive bladder appears to differ by gender: Results from the Boston Area Community Health survey. J Urol 2011; 185: 955-63.;

[4] Patra PB, Patra S. Sex differences in the physiology and pharmacology of the lower urinary tract. Curr Urol 2013; 6: 179-88.

[5] Choo MS, Ku JH, Lee JB, et al. Cross-cultural differences for adapting overactive bladder symptoms: Results of an epidemiologic survey in Korea. World J Uro 2007; 25: 505-11.

[6] Coyne KS, Sexton CC, Vats V, et al. National community prevalence of overactive bladder in the United States stratified by sex and age. Urology 2011; 77: 1081-7.

[7] Gammie A, Clarkson B, Constantinou C, et al. International continence society guidelines on urodynamic equipment performance. Neurourol. Urodyn. 2014; 33: 370-379.

[8] Digesu GA, Khullar V, Cardozo L, Salvatore S. Overactive bladder symptoms: do we need urodynamics? Neurourol Urodyn. 2003; 22: 105-8. Erratum in: Neurourol Urodyn. 2003; 22: 356 .

[9] Gormley, EA; Lightner, DJ; Faraday, M; Vasavada, SP (May 2015). "Diagnosis and Treatment of Overactive Bladder (NonNeurogenic) in Adults: AUA/SUFU Guideline Amendment." The Journal of Urology. 193 (5): 1572-80.

[10] Farzeen Firoozi. Interpretation of Basic and Advanced Urodynamics. Springer International Publishing Switzerland; 2017. p. 134. 